\title{
Peculiarities of near-electrode relaxation processes in the polyethylene melt filled with graphite and carbon black
}

\author{
Ya.A. Kuryptya ${ }^{1}$, B.M. Savchenko ${ }^{1}$, O.V. Kovalchuk ${ }^{1}$, T.M. Kovalchuk ${ }^{2}$, T.S. Shostak \\ ${ }^{1}$ Kyiv National University of Technologies and Design, Kyiv, Ukraine \\ ${ }^{2}$ V. Lashkaryov Institute of Semiconductor Physics, National Academy of Sciences of Ukraine, Kyiv, Ukraine \\ E-mail:akoval@knutd.com.ua
}

\begin{abstract}
By using the oscilloscope method within the frequency range 10 to $10^{6} \mathrm{~Hz}$ at the temperature $492.1 \mathrm{~K}$ and pressure $11.31 \mathrm{MPa}$ at the output of the single-screw extruder, the dielectric properties of the composite melt - linear low-density polyethylene $+20 \mathrm{wt} . \%$ of graphite $+10 \mathrm{wt} . \%$ of carbon black - have been investigated. It has been shown that in the whole frequency range, near-electrode processes significantly effect on charge transfer. For the frequencies less than $100 \mathrm{~Hz}$, in the near-electrode area the conductance is provided with hopping movement of electrons. For the frequencies over $100 \mathrm{~Hz}$, charge transfer occurs through the ion movement. It has been shown that the transfer of ions in the near-electrode area can be described using the relaxation process that, unlike the vast majority of processes described in the publications, meets the wellknown relations only in terms of the complex conductivity. It has been shown that, in the terms of the complex conductivity, this relaxation process can be approximated with Debye equation, as well as it has been estimated the relaxation time $(8.5 \mathrm{~ms})$ for this process. According to the parameters included in the Debye equation, it has been estimated the thickness of the near-electrode area $(17 \mu \mathrm{m})$ and shown that it practically coincides with the sizes of graphite particles.
\end{abstract}

Keywords: polyethylene, graphite, carbon black, conductance, dielectric properties, nearelectrode relaxation process, single-screw extruder.

Manuscript received 12.04.16; revised version received 14.07.16; accepted for publication 13.09.16; published online 04.10.16.

\section{Introduction}

Currently, polymer composites are widely used in various fields of technology. Therefore, to expand their functionality, studies are performed using different types of polymers and fillers [1-8]. In recent years for such studies, the use of nanoparticles is typical [9-10].

It is clear that the properties of polymer composites will essentially depend on the polymer material. For specific tasks, it is not always necessary to look for new materials, but polymers long been synthesized can be used. These polymers include polyethylene [11-12]. The interest to this material will increase significantly, as most recently it was disclosed broad applicability of polyethylene in nanoporous form for making clothes, which by its parameters (thermal conductivity and moisture permeability) exceeds the natural material such as cotton [13]. Beside polyethylene as a separate material, it is important to create composites based on polyethylene with the desired properties. The necessary properties can be better provided with polyethylene or composites based on it, if to expand the conditions of controlling the material parameters directly in the technological process.

For the majority of the works analyzed by us from the viewpoint of properties inherent to composites based on polyethylene, it is typical that the physical properties of these materials are investigated after the polymerization process. This made it impossible to intervene into the process, especially when the quality of 
polyethylene or filler does not meet the required parameters in accordance with the technological chart of the process. When manufacturing the composite materials, it is very important to provide uniformity of distribution of impurities over the whole polymer bulk. It can be definitely evaluated when studying the dielectric properties of material during the technological process. To make the task of research closer to real technological conditions in industry, it is important to use several components of different properties for manufacturing composites based on polyethylene.

One of the main methods for preparation of homogeneous polymers or composites based on them is extrusion. Therefore, the purpose of this work was to study dielectric properties of melt of polyethylene composite with graphite filler and carbon black at the final stage of manufacturing in the extruder.

\section{Materials and methods}

To produce the composite powder, we used powdered linear low-density polyethylene. The concentration of polyethylene in the composite was $70 \mathrm{wt} . \%$. The main (by weight) impurity was graphite (colloid specimen, grade $\mathrm{C}-1$, carbon content of $99.3 \%$, made in Ukraine). The form of graphite particles was like pebbles: spherical, elongated. The particle size was within 2.3 to $50 \mu \mathrm{m}$. The graphite concentration in the composite was 20 wt. $\%$.

One more impurity used for creating the composite was carbon black (PUREBLACK SCD-205 (USA)). The average size of the carbon black particles was equal to $42 \mathrm{~nm}$, and specific surface area is $50 \mathrm{~m}^{2} / \mathrm{g}$. The concentration of graphite in the composite was $10 \mathrm{wt} . \%$.

We produced the composite based on polymer, graphite and carbon black by using single-screw extruder with the diameter $25 \mathrm{~mm}$. The main elements of the extruder are schematically depicted in Fig. 1.
A cell for measuring the resistance $R$ and capacitance $C$ were mounted into the extruder head. Its construction is shown in Fig. 2. The distance between electrodes was $d=1 \mathrm{~mm}$. The area of the electrodes was equal to $S=5.72 \mathrm{~mm}^{2}$.

The dielectric properties of the obtained sandwich cells were studied within the frequency range 10 to $10^{6} \mathrm{~Hz}$ at the temperature $293 \mathrm{~K}$ by using the oscilloscopic method [14]. Oscillograms were photographed using a digital camera, and then their treatment was held. This made it possible to significantly speed up the measurement process, which was very important for these studies.

The amplitude of the measuring signal with sinusoidal shape was $0.4 \mathrm{~V}$. Assuming that the equivalent circuit of the measuring cell is connected in parallel to the resistance and capacitor, we determined the value of resistance $R$ and capacitance $C$ of the samples at different frequencies.

The study was performed using the method developed by us for measuring electrical properties of the polymer melt during its processing as described in the patent [15]. The method is to measure the dielectric properties of the polymer in the molten state at processing temperature by using two electrodes in the form of thin rods that are placed in the extruder after the dosing zone. The mixture of composite components is loaded to the extruder hopper after its transition to the viscous-flow state and homogenization, at the end of the third zone of the extruder, in the mounted adapter the dielectric parameters being measured.

Our research has shown that during the first extrusion and partly during the second one, the meterage has changed over time. Therefore, the study of composites within the frequency range was performed starting from the third extrusion.

In this work, the data were analyzed for seventh extrusion. Technological process parameters for this extrusion are given in Table.

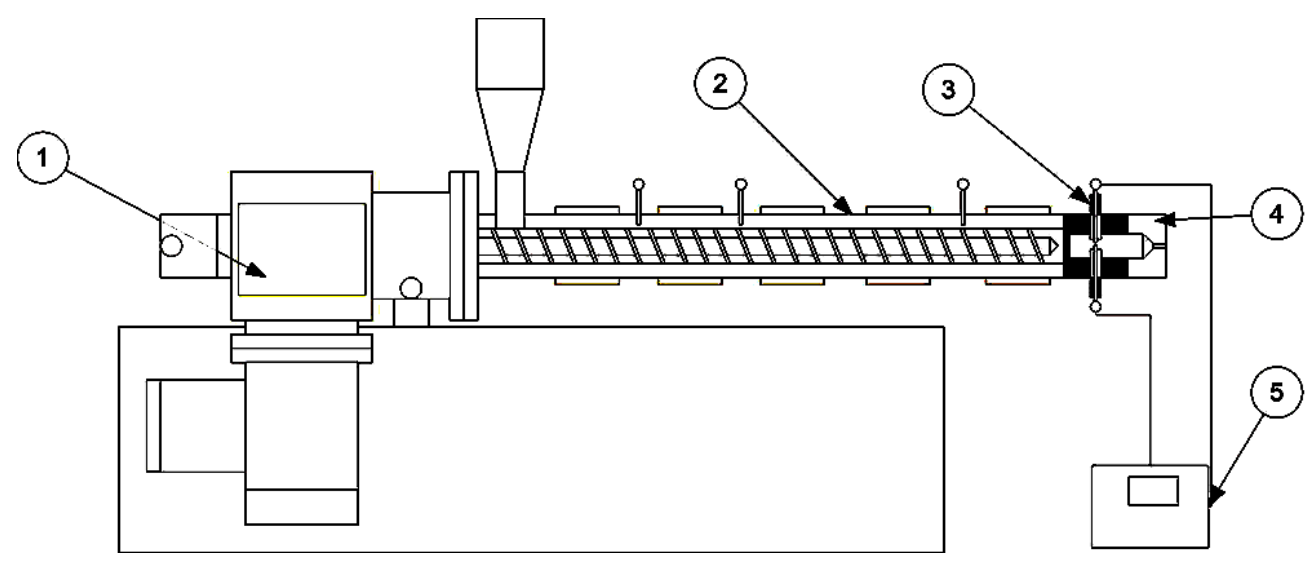

Fig. 1. Scheme for manufacturing and research of dielectric properties of polyethylene melt with impurities of graphite and carbon black in extruder: 1 -drive of extruder; 2 - extruder, 3 - adapter for measuring the electrical conduction of the polymer melt with electrodes for measurement; 4 - heads of the extruder with forming filament extrusion device, 5 - device for measuring the resistance and capacitance by using the oscilloscope method within the wide frequency range. 

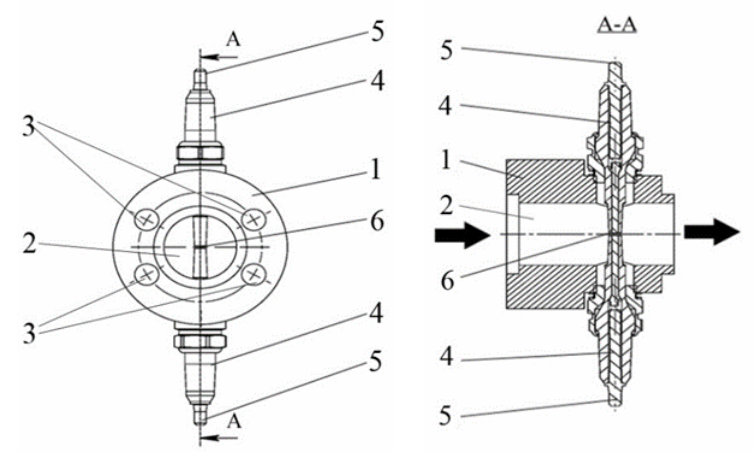

Fig. 2. The structure of the cell to study the dielectric properties of polyethylene melt with graphite and carbon black. 1 - adapter, 2 - internal channel of adapter, 3 - mounting holes of adapter, 4 - spark plugs built into the adapter, 5 - electrical contacts of spark plugs, 6 - gap between the electrodes in the inner channel of adapter.

Table. Parameters of the technological process for the seventh extrusion of polyethylene composite with graphite and carbon black.

\begin{tabular}{|c|c|c|c|c|c|}
\hline 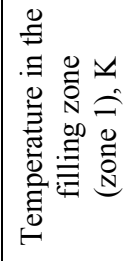 & 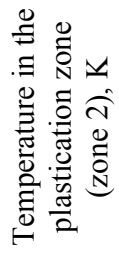 & 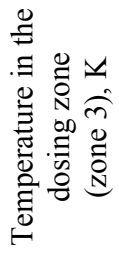 & 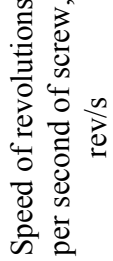 & 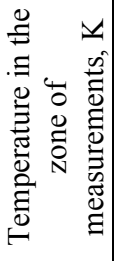 & 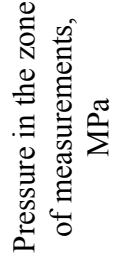 \\
\hline 437 & 473 & 483 & 8.49 & 492.1 & 11.31 \\
\hline
\end{tabular}

\section{Results and discussion}

Our analysis of the measured frequency dependences of resistance and capacitance has shown that dispersion of $R$ and $C$ is observed almost in the whole range of frequencies $f$. It is known [16-18] that analysis of the frequency dependences of $R$ and $C$ is performed using the dielectric spectroscopy methods. In dielectric spectroscopy, the analysis of these dependences is mainly made in the terms of complex permittivity $\varepsilon^{*}$ :

$\varepsilon^{*}=\varepsilon^{\prime}-i \varepsilon^{\prime \prime}$,

where $\varepsilon^{\prime}$ - real and $\varepsilon^{\prime \prime}$ - imaginary components of complex permittivity.

The frequency dependences $\varepsilon^{\prime}(1)$ and $\varepsilon^{\prime \prime}(2)$ of polyethylene melt with impurities of graphite and carbon black are shown in Fig. 3.

As follows from the analysis of the data, the value $\varepsilon^{\prime}$ is higher than 10 for $f<10^{5} \mathrm{~Hz}$ and is equal to 1000 at the frequencies near $f \approx 10^{3} \mathrm{~Hz}$. As we have shown in $[19,20]$, these large $\varepsilon^{\prime}$ quantities are caused by inhomogeneous distribution of the electric field in the sample due to the impact of near-electrode processes. Therefore, these large $\varepsilon^{\prime}$ quantities can be cosidered as the effective ones. In $[19,20]$, we have also shown as the dispersion of $\varepsilon^{\prime}$ and $\varepsilon^{\prime \prime}$, which is caused by the influence of near-electrode processes, is described by the same relations that the dispersion in the bulk of samples (Debye dispersion, Cole-Cole dispersion, etc.).

The analysis of data in Fig. 3 shows that, in the terms of the complex permittivity, dispersion of $\varepsilon^{\prime}$ and $\varepsilon^{\prime \prime}$ is not described by any of the known laws.

As shown in [18], dispersion of $R$ and $C$ on the frequency of measuring signal can be also analyzed in the terms of the complex conductance:

$\sigma^{*}=\sigma^{\prime}+i \sigma^{\prime \prime}$,

where $\sigma^{\prime}$ - real and $\sigma^{\prime \prime}$ - imaginary components of the complex conductance. In this case,

$\sigma^{\prime}=\varepsilon_{0} \omega \varepsilon^{\prime \prime}$

and

$\sigma^{\prime \prime}=\varepsilon_{0} \omega \varepsilon^{\prime}$,

where $\varepsilon_{0}$ is the electric constant, and $\omega=2 \pi f$ is the cyclic frequency.

The frequency dependences $\sigma^{\prime}(1)$ and $\sigma^{\prime \prime}(2)$ for polyethylene melt with the impurities of graphite and carbon black are shown in Fig. 4.

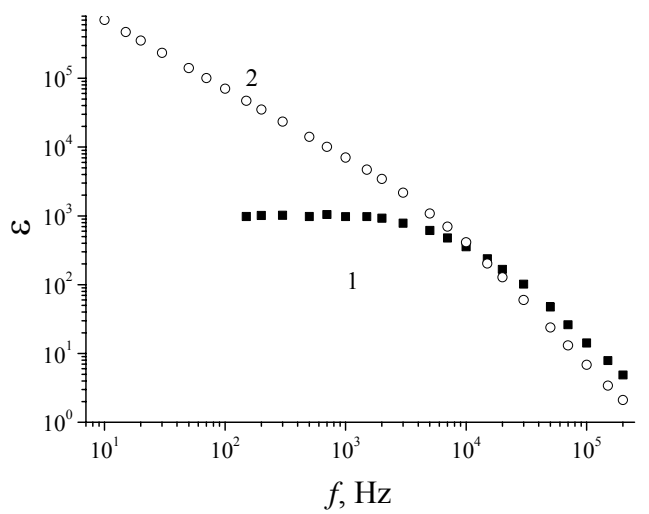

Fig. 3. Frequency dependences of the real $\varepsilon^{\prime}(1)$ and imaginary $\varepsilon^{\prime \prime}(2)$ components of complex permittivity of polyethylene melt with impurities of graphite and carbon black. The melt temperature is $219.1{ }^{\circ} \mathrm{C}$, the pressure in the measuring area is 11.31 MPa.

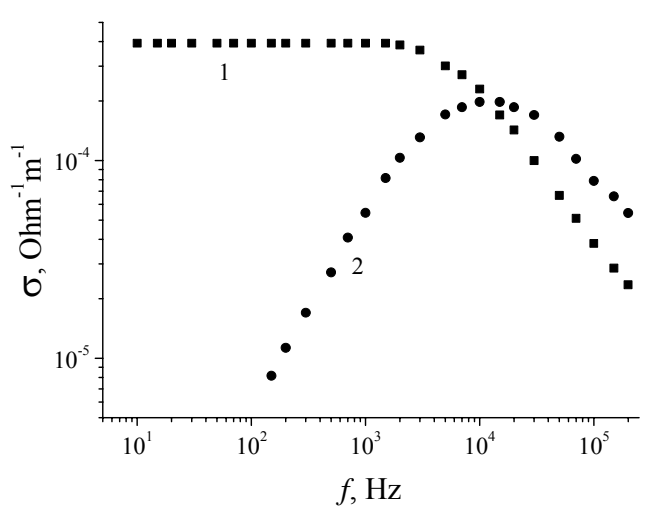

Fig. 4. Frequency dependences of the real $\sigma^{\prime}(1)$ and imaginary $\sigma^{\prime \prime}(2)$ components of complex conductance of polyethylene melt with impurities of graphite and carbon black. The melt temperature is $492.1{ }^{\circ} \mathrm{C}$, the pressure in the measuring area is $11.31 \mathrm{MPa}$. 


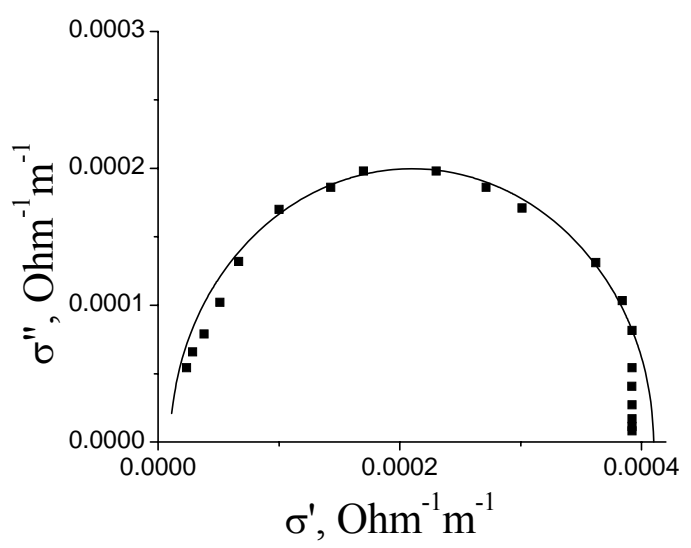

Fig. 5. Cole-Cole diagram (dependence $\sigma^{\prime \prime}\left(\sigma^{\prime}\right)$ ) for the data presented in Fig. 4.

Unlike the data presented in Fig. 3, the plots in Fig. 4 correspond to the known laws for relaxation processes. To assertain the type of relaxation process in accord with the data shown in Fig. 4, we constructed and analyzed the Cole-Cole diagram. In the case of complex conductance, it corresponds to the dependence $\sigma^{\prime}\left(\sigma^{\prime \prime}\right)$ that is graphically depicted in Fig. 5.

As follows from Fig. 5, the dependence $\sigma^{\prime}\left(\sigma^{\prime \prime}\right)$ can be approximated by a semicircle. According to the theory of relaxation processes [16-18], this dispersion in the terms of the complex conductance can be considered as the Debye dispersion and described by the relation

$\sigma^{*}=\sigma_{\infty}+\frac{\sigma_{s}-\sigma_{\infty}}{1+i \omega t}$,

$\sigma_{\infty}$ is the conductance value at $f=\infty, \sigma_{s}$ - conductance value at $f=0$, and $\tau$ - relaxation time. Estimations of the $\sigma_{\infty}$ and $\sigma_{s}$ values from the Cole-Cole diagrams are as follows: $\sigma_{\infty}=1.4 \cdot 10^{-5}$ and $\sigma_{s}=4.1 \cdot 10^{-4} \mathrm{Ohm}^{-1} \mathrm{~m}^{-1}$. We estimate the value of the relaxation time: $\tau=8.5 \mathrm{~ms}$.

Being based on the permittivity $\varepsilon_{\infty}$ value at $f=\infty$ and $\varepsilon_{s}$ one at $f=0$ in the work [19], from the expression

$l=d \frac{\varepsilon_{\infty}}{2 \varepsilon_{s}}$

we have first estimated (based on dielectric spectroscopy data) the thickness of near-electrode area $l$, in which the relaxation process occurs.

Our analysis indicates that, in the case of considerating the causes of dispersion of $R$ and $C$ on the frequency in the terms of complex conductance, for estimation of the thickness of near-electrode area one can use the formula similar to the equation (6), namely:

$l=d \frac{\sigma_{\infty}}{2 \sigma_{s}}$.

The thickness of the near-electrode area evaluated on the basis of the relation (7) is $17 \mu \mathrm{m}$. From the comparison of the $l$ value with particle sizes of graphite and carbon black, one can assume that blocking the exchange of charge on the interface 'melt of composite electrode' is caused by graphite, the particle size of which is comparable with the $l$ value. It may be also suggested that for the frequencies $f>10^{2} \mathrm{~Hz}$ the electrical current in the melt is provided by the transfer of ions. These mobile ions can be both separate uncontrolled ions that may be contained in each component of the composite and ions adsorbed on the surface of carbon black nanoparticles. Much larger particles of graphite can limit the process of interaction between these ions and electrodes. As a result, the electric field is mainly applied to the nearelectrode areas.

As it follows from Fig. 5, at the frequencies $f<$ $10^{2} \mathrm{~Hz}$ (right wing in the Cole-Cole diagram) there is a significant deviation from the circle. It can be explained by the presence of the electron component of conductance, except the mechanism of ionic charge transfer through the melt composite. For the case of a disordered structure that is the composite under study, these charge transfer can not be considered on the basis of the band model developed for the ordered solids, but can be considered as hopping transfer of electrons in the model of disordered structures. Certain time is necessary to make the hop. Therefore, at the frequencies higher than the reciprocal value of this time, the charge transfer efficiency by using electrons becomes very low. Taking into accout that the deviation of the Debye law (equation (5)) begins at the frequencies $100 \mathrm{~Hz}$, we can assume that for hopping of electron from one article of carbon black to another, it takes time no less than $10 \mathrm{~ms}$.

\section{Results and conclusions}

By using the oscilloscope method within the frequency range 10 to $10^{6} \mathrm{~Hz}$ at the temperature $492.1 \mathrm{~K}$ and pressure $11.31 \mathrm{MPa}$ at the output of the single-screw extruder, dielectric properties of the composite consisting of linear low-density polyethylene, $20 \mathrm{wt} . \%$ of graphite and $10 \mathrm{wt} . \%$ of carbon black have been investigated. It has been shown that in the whole frequency range, dispersion of $R$ and $C$ on the frequency is observed. From the analysis of the frequency dependences of components of complex permittivity, it has been concluded that for the whole frequency range the electric field is mainly applied to the near-electrode areas.

It has been shown that the relaxation process, by using which dispersion of resistance and capacitance can be described, cannot be approximated being based on the terms of the complex dielectric permittivity, because it does not correspond to any of already described in publications (Debye dispersion, Cole-Cole dispersion, etc.). The detailed analysis of the relaxation process determined experimentally has shown that it can be described by the Debye equation, but in the terms of the complex conductance. Estimated have been the value of the relaxation time $(8.5 \mathrm{~ms})$ and the thickness of near-electrode area $(17 \mu \mathrm{m})$, in which the relaxation process occurs. 
Since the thickness of the near-electrode area matches the sizes of the graphite particles, it was assumed that blocking the exchange of charge between charge carriers and electrodes occurs due to the graphite particles adsorbed on the electrode surface. It has been also assumed that the Debye equation describes the transfer of ions of uncontrolled impurities of both individual particles and those bound with nanoparticles of carbon black.

It has been shown that deviation from the Debye law for the frequencies below $100 \mathrm{~Hz}$ can be explained by contribution of the electron component of conductance into charge transfer in the near-electrode area. For disordered structure, which is polymer melt, this charge transfer can occur hoppingly. Since the electron mechanism of transfer becomes effective for the frequencies below $100 \mathrm{~Hz}$, it gives the value of time not less than $10 \mathrm{~ms}$ to transfer electrons.

\section{Acknowledgements}

The work shows the results of investigation in accord with the scientic program "Development of an integrated method of forming the ergonomic and functional and operational characteristics of footwear, haberdashery products, special (for servicemen) and domestic purposes" (16/04/52 ДБ) Ministry of Education and Science of Ukraine.

\section{References}

1. M. Amin, M. Ali, Polymer nanocomposites for high voltage outdoor insulation applications // Rev. Adv. Mater. Sci., 40, p. 276-294 (2015).

2. B.S. Rana, Effect of graphite filler on dielectric properties of polymer composite // Special Issue on International Journal of Recent Advances in Engineering \& Technology (IJRAET) Vol. 4, Iss. 5, 2016, For National Conference on "Recent Innovations in Engineering and Management Sciences" (RIEMS-2016) Modern Engineering \& Management Studies Balasore, ODISHA, ISSN (Online): 2347-2812, March 20, 2016.

3. M. Matsuo, Electric, Dielectric and Magnetic Properties of Polymer and Carbon Fillers // International Workshop on Advanced Polymer Science and Turbulent Drag Reduction, March 10 20, 2008 .

4. E. Tuncer, Y.V. Serdyuk, S.M. Gubanski, Dielectric mixtures: Electrical properties and modeling // Transactions on Dielectrics and Electrical Insulation, 9, No.5, p. 809-828 (2002).

5. W. Zheng, Sh.-Ch. Wong, Electrical conductivity and dielectric properties of PMMA/expanded graphite composites // Composites Sci. and Technol. 63, p. 225-235 (2003).

6. H. Oxfall, G. Ariu, T. Gkourmpis, R.W. Rychwalski, M. Rigdahl, Effect of carbon black on electrical and rheological properties of graphite nanoplatelets/poly(ethylene-butylacrylate) composites // eXPRESS Polymer Letters, 9, No.1, p. 66-76 (2015).

7. C. Calberg, S. Blacher, F. Gubbels, F. Brouers, R. Deltour and R. Jerome, Electrical and dielectric properties of carbon black filled co-continuous two-phase polymer blends // J. Phys. D: Appl. Phys. 32 p. 1517-1525 (1999).

8. M. Pluta, M. Alexandre, S. Blacher, P. Dubois, R. Jerome, Metallocene-catalyzed polymerization of ethylene in the presence of graphite. II. Structure and electrical properties of the composites // Polymer, 42, No. 22, p. 9293-9300 (2001).

9. L. He, S.-C. Tjong, Electrical behavior and positive temperature coefficient effect of graphene/polyvinylidene fluoride composites containing silver nanowires // Nanoscale Res. Lett. 9, p. 375 (2014).

10. P.H.S.L. Coelho, M.S. Marchesin, A.R. Morales, J.R. Bartoli, Electrical Percolation, Morphological and Dispersion Properties of MWCNT/PMMA Nanocomposites // Materials Res. 17 (Suppl. 1), p.127-132 (2014).

11. K.-B. Yoon, G. H. Lee, W. Y. Choi, and D. Lee, Electrical properties of non-crosslinked polyethylene/syndiotactic polystyrene composites filled with carbon black // Polymer J., 39, No. 11, p. 1143-1149 (2007).

12. G. Pavoski, T. Maraschin, M.A. Milani, D.S.Azambuja, R. Quijada, C.S. Moura, N.S. Basso, G. B. Galland, Polyethylene/reduced graphite oxide nanocomposites with improved morphology and conductivity // Polymer, 81, p.7986 (2015).

13. http://www.azom.com/news.aspx?newsID $=46313$

14. A.J. Twarowski, A.C. Albrecht, Depletion layer in organic films: Low frequency measurements in polycrystalline tetracene // J. Chem. Phys. 70, p. 2255-2263 (1979).

15. Patent № 105113 Ukraine, CI G01N 27/02. Method of measuring the electrical properties of polymer / B.M. Savchenko, N.V. Sova, Ya.A. Kuryptya, O.O. Sleptsov, I.L. Sleptsova. - № 201507226; applied from 20.07.2015; publ. 10.03.2016, bul. №5.

16. F. Kremer, A. Schönhals, Broadband Dielectric Spectroscopy. Springer, 2003.

17. W. Haase, S. Wrobel, Relaxation Phenomena: Liquid Crystals, Magnetic Systems, Polymers, High-Tc. Springer, 2003.

18. T.L. Chelidze, A.I. Derevianko, O.D. Kurilenko, Electrical Spectroscopy of Heterogeneous Systems. Naukova Dumka, Kiev, 1977 (in Russian).

19. A.V. Koval'chuk, Low and infra-low dielectric spectroscopy liquid crystal-solid state interface. Sliding layers // Ukr. J. Phys. 41(10), p. 991-998 (1996).

20. A.V. Koval'chuk, Relaxation processes and charge transport across liquid crystal-electrode interface // J. Phys.: Condensed Matter, 13, p. 10333-10345 (2001). 